ESAIM: PROCEEDINGS, October 2011, Vol. 32, p. 195-210

E. Cancès, N. Crouseilles, H. Guillard, B. Nkonga, and E. Sonnendrücker, Editors

\title{
CONSERVATIVE NUMERICAL METHODS FOR A TWO-TEMPERATURE RESISTIVE MHD MODEL WITH SELF-GENERATED MAGNETIC FIELD TERM
}

\author{
Marc WolfF ${ }^{1}$, Stéphane JaOuen $^{1}$ And Lise-Marie Imbert-GÉrard ${ }^{2}$
}

\begin{abstract}
We propose numerical methods on Cartesian meshes for solving the 2-D axisymmetric two-temperature resistivive magnetohydrodynamics equations with self-generated magnetic field and Braginskii's [1] closures. These rely on a splitting of the complete system in several subsystems according to the nature of the underlying mathematical operator. The hyperbolic part is solved using conservative high-order dimensionally split Lagrange-remap schemes whereas semi-implicit diffusion operators have been developed for the thermal and resistive conduction equations. Source terms are treated explictly. Numerical results on the deceleration phase of an ICF implosion test problem are proposed, a benchmark which was initially proposed in [2].

Résumé. Nous proposons dans cet article des méthodes numériques pour les équations de la magnétohydrodynamique résistive à deux températures avec champ magnétique auto-généré et relations de fermeture de Braginskii [1] en géométrie 2-D axisymétrique sur maillage cartésien. Celles-ci sont basées sur une décomposition du système complet selon la nature des opérateurs mathématiques sous-jacents. La partie hyperbolique est résolue par des schémas conservatifs Lagrange-projection d'ordre élevé en directions alternées tandis que des opérateurs de diffusion semi-implicites ont été développés pour les équations de conduction thermique et résistive. Les termes sources sont traités de manière explicite. Des résultats numériques sur un cas-test simulant la phase de décélération d'une implosion de capsule FCI sont proposés, ce benchmark ayant été initialement présenté dans [2].
\end{abstract}

\section{INTRODUCTION}

The accurate simulation of inertial confinement fusion (ICF) is an important topic for plasma physics scientists. Indeed, it is well-known that, due to the non-uniformity of the laser irradiation and/or rugosity on the shell's surface, instabilities may develop and lead to the generation of magnetic field [3]. Depending on their intensity, such MHD source terms may contribute to notably reduce thermal diffusion, modify the temperature in the neighbouring of the hot spot and charged particle transport, thus modifying the ignition conditions. It therefore seems important to follow the evolution of the magnetic field in addition to the usual hydrodynamic treatment in ICF simulations.

We propose in this paper a two-temperature resistive MHD model with self-generated magnetic field terms for direct drive ICF. The implosion of an ICF shell being a spherically symmetric phenomenom, our simulations will be performed in the 2-D axisymmetric geometry (numerical methods being though developed for both planar and axisymmetric cases). This choice seems to be a good compromise since it will allow us to consider an

1 CEA, DAM, DIF, F-91297 Arpajon, France. e-mail: marc.wolff@cea.fr \& stephane.jaouen@cea.fr

${ }^{2}$ Laboratoire JLL, UPMC, 4 place Jussieu, F-75005 Paris, France. e-mail: imbert@ann.jussieu.fr

(C) EDP Sciences, SMAI 2011 
initial perturbation of the interface in the $(z, r)$ plane and to follow its evolution without resorting to expensive $3-\mathrm{D}$ computations. In this context, self-generated magnetic field terms may only appear along the $\theta$ direction (see section 2.3), namely along the direction that is perpendicular to the computational domain. Note that we therefore automatically guarantee that $\nabla \cdot \mathbf{B}=0$.

The outline of the paper is the following. Section 1 briefly describes the considered equations and the underlying physical assumptions. In section 2 we detail numerical methods that have been developed to discretize these equations, which will be split according to the nature of the underlying operator. Validation test problems for the diffusion operator are proposed in section 3. In section 4 we present a relevant two-temperature benchmark for simulating the deceleration phase of an ICF target in the direct drive context, a test problem which has been initially proposed in [2]. In section 4.2 numerical results without magnetic field in the $1 \mathrm{~T}$ case are compared to those obtained with the 1-D Lagrangian perturbation code (LPC) in spherical geometry [2,4], whereas in section 4.3, 2-D results on the whole system - with/without Legendre perturbed gas/shell interface - are proposed.

\section{MOdel DESCRIPTION}

\subsection{Notations}

For each species $\alpha$ (namely ions and electrons, denoted by $i$ and $e$ subscripts in the sequel), the variables $\rho_{\alpha}$, $n_{\alpha}$ respectively denote the mass and particle densities, $\mathbf{u}_{\alpha}$ the velocity, $p_{\alpha}$ the pressure, $T_{\alpha}$ the temperature $\epsilon_{\alpha}$ the internal energy and $m_{\alpha}$, the mass of particle $\alpha$. Since we only deal with perfect gases in this study, the internal energy is given by the following relation:

$$
\epsilon_{\alpha}=C_{v_{\alpha}} T_{\alpha}=\frac{3 k_{B}}{2 m_{\alpha}} T_{\alpha}
$$

where $k_{B}$ denotes the Boltzmann constant. In the sequel, we will also use $c$, the light velocity and $e$ the charge of an electron. In Braginskii's closure relations [1] that will be used here, the resistivity $\overline{\bar{\rho}}$, the thermoelectric $\overline{\bar{\beta}}$ and the conductivy $\overline{\overline{\kappa_{i}}}$ and $\overline{\overline{\kappa_{e}}}$ tensors are expressed in $(\|, \perp, \wedge)$ components that refer to the direction of the magnetic field. Let $\mathbf{h}$ be a unit vector along this direction, these are defined as

$$
\begin{aligned}
\mathbf{u}_{\|} & =(\mathbf{h} \cdot \mathbf{u}) \mathbf{h}, \\
\mathbf{u}_{\wedge} & =\mathbf{h} \wedge \mathbf{u}, \\
\mathbf{u}_{\perp} & =(\mathbf{h} \wedge \mathbf{u}) \wedge \mathbf{h} .
\end{aligned}
$$

We also introduce the following notation:

$$
\overline{\bar{T}} \cdot \mathbf{u}=T^{\|} \mathbf{u}_{\|}+T^{\wedge} \mathbf{u}_{\wedge}+T^{\perp} \mathbf{u}_{\perp}=\left(T^{\perp} u_{z}-T^{\wedge} u_{r}\right) \mathbf{e}_{z}+\left(T^{\perp} u_{r}+T^{\wedge} u_{z}\right) \mathbf{e}_{r} \text { in our case since } \mathbf{h}=\mathbf{e}_{\theta} .
$$

\subsection{Physical assumptions}

Our model has been built under two physical assumptions. First, the displacement current $\partial_{t} \mathbf{E}$ is neglected. Let $\mathbf{J}$ denote the current density. The Ampère-Maxwell equation $\mathbf{J}=\frac{c}{\mu} \nabla \times \mathbf{B}-\frac{1}{\mu} \partial_{t} \mathbf{E}$ therefore leads to:

$$
\mathbf{J}=\frac{c}{\mu} \nabla \times \mathbf{B}
$$

We also assume that the plasma is locally neutral, so that the ionic and electronic particle densities satisfy the following relation ( $Z$ being the electric charge):

$$
n_{i} Z_{i}+n_{e} Z_{e}=0 \quad \text { i.e. } \quad n_{i} Z_{i}=n_{e} \quad \text { since } Z_{e}=-1 .
$$




\subsection{Model equations}

The model equations given hereafter mainly rely on the Vlasov-Maxwell equations in the limit $m_{e} \ll m_{i}$ whose complete derivation can be found in [5]. Contrarily to [5], we neglect the Hall and Nernst effects contributions in this paper. Defining $\rho=\rho_{i}+\rho_{e}$ the total density, $p=p_{i}+p_{e}$ the total gas pressure and $\mathbf{u}=\left(\rho_{i} \mathbf{u}_{i}+\rho_{e} \mathbf{u}_{e}\right) / \rho$ the mean velocity, the mass continuity and momentum equations are the classical ideal MHD equations:

$$
\begin{aligned}
& \partial_{t} \rho+\nabla \cdot(\rho \mathbf{u})=0, \\
& \partial_{t}(\rho \mathbf{u})+\nabla \cdot\left(\rho \mathbf{u} \otimes \mathbf{u}+\left(p+\frac{B^{2}}{2 \mu}\right) \underline{\mathbf{I}}-\frac{\mathbf{B} \otimes \mathbf{B}}{\mu}\right)=0 .
\end{aligned}
$$

The electronic and ionic internal energy equations write

$$
\begin{aligned}
& \partial_{t}\left(\rho_{e} \epsilon_{e}\right)+\nabla \cdot\left(\rho_{e} \epsilon_{e} \mathbf{u}\right)+p_{e} \nabla \cdot \mathbf{u}+\nabla \cdot \mathbf{q}_{e}=Q_{e i}, \\
& \partial_{t}\left(\rho_{i} \epsilon_{i}\right)+\nabla \cdot\left(\rho_{i} \epsilon_{i} \mathbf{u}\right)+p_{i} \nabla \cdot \mathbf{u}+\nabla \cdot \mathbf{q}_{i}=-Q_{e i},
\end{aligned}
$$

the magnetic field equation being given by

$$
\partial_{t} \mathbf{B}+\nabla \cdot(\mathbf{u} \otimes \mathbf{B}-\mathbf{B} \otimes \mathbf{u})=c \nabla \times\left(\mathbf{S}_{\text {self }}-\mathbf{R}_{e i}\right),
$$

with the self-generated magnetic field source terms defined by

$$
\mathbf{S}_{\mathrm{self}}=\frac{1}{e n_{e}} \nabla p_{e} .
$$

To close these equations, we still have to define the ionic and electronic heat fluxes $\mathbf{q}_{i}$ and $\mathbf{q}_{e}$ and the collision kernels $Q_{e i}$ and $\mathbf{R}_{e i}$. Our closure relations, taken from the Braginskii's model [1], are given by

$$
\begin{aligned}
\mathbf{R}_{e i} & =\overline{\bar{\rho}} \cdot \mathbf{J}, \\
Q_{e i} & =\mathbf{R}_{e i} \cdot \mathbf{J}+3 \frac{m_{e}}{m_{i}} n_{e} \nu_{e i} k_{B}\left(T_{i}-T_{e}\right), \\
\mathbf{q}_{e} & =-k_{B}\left(\overline{\overline{\kappa_{e}}} \cdot \nabla T_{e}\right)-\frac{k_{B} T_{e}}{e}(\overline{\bar{\beta}} \cdot \mathbf{J}), \\
\mathbf{q}_{i} & =-k_{B}\left(\overline{\overline{\kappa_{i}}} \cdot \nabla T_{i}\right),
\end{aligned}
$$

where $\nu_{e i}$ denotes the collision frequency between ions and electrons. Detailed expressions of $(\|, \perp, \wedge)$ tensors components are given in [1].

These equations will be particularized for the 2-D axisymmetric geometry in the sequel. In this context, if B is initially zero, one easily shows that $B_{r}=B_{z}=0$ for all times, so that we will only have to deal with orthogonal MHD equations.

\section{Numerical methods And general Splitting STRATEGy}

The complete model equations (4-8) are treated separately according to the mathematical nature of each operator thanks to a first-order Godunov splitting. We present in this section numerical methods that have been developed for the resulting subsystems. These are listed in Table 1 together with the time scheme used. An explicit scheme being used to solve the hyperbolic part, the time step is therefore constrained by a classical CFL condition. To preserve this time step for parabolic operators without losing stability, the thermal and resistive diffusion subsystems are treated semi-implicitly. We should theoretically do so for the source terms system but such an approach would be too costly due to the complexity of its equations. Practically, it appears 
that the source terms' contribution is weak compared to the previously mentioned systems, so that it can be solved with an explicit time scheme.

\begin{tabular}{|c|c|c|}
\hline Subsystem & Mathematical operator & Time scheme \\
\hline orthogonal MHD & hyperbolic & explicit \\
thermal diffusion & parabolic & semi-implicit \\
resistive diffusion & parabolic & semi-implicit \\
source terms & source terms & explicit \\
\hline
\end{tabular}

TABLE 1. Considered subsystems.

\subsection{Hyperbolic operator: the orthogonal MHD system}

We first consider the orthogonal MHD equations, namely (4-8) in the specific case $\mathbf{B}=B$ e $\theta$, without righthand side nor heat fluxes. Numerical schemes are developed here on the conservative form of this system which is derived by replacing (6-7) by equations satisfied by total energy $\rho e$ and electronic entropy $S_{e}$ (see [6,7] for theoretical justifications about this variable change) given by

$$
\begin{aligned}
\rho e & =\rho_{i} \epsilon_{i}+\rho_{e} \epsilon_{e}+\frac{1}{2} \rho \mathbf{u}^{2}+\frac{B^{2}}{2 \mu} \\
S_{e} & =\ln \left(\left(\rho_{e} \epsilon_{e}\right) \rho^{-\gamma_{e}}\right)
\end{aligned}
$$

A few manipulations therefore lead to the following conservative system, with $P=p+B^{2} /(2 \mu)$ the total pressure:

$$
\partial_{t}\left(\begin{array}{c}
\rho \\
\rho \mathbf{u} \\
\rho e \\
\rho_{e} S_{e} \\
B
\end{array}\right)+\nabla \cdot\left(\begin{array}{c}
\rho \mathbf{u} \\
\rho \mathbf{u} \otimes \mathbf{u}+P \underline{\mathbf{I}}-\frac{\mathbf{B} \otimes \mathbf{B}}{\mu} \\
(\rho e+P) \mathbf{u} \\
\rho_{e} S_{e} \mathbf{u} \\
\mathbf{u} \otimes \mathbf{B}-\mathbf{B} \otimes \mathbf{u}
\end{array}\right)=\mathbf{0} .
$$

Numerically, system (10) is solved using a high-order dimensionally split Lagrange-remap scheme - formulated in total energy - that was initially developed for planar hydrodynamics [8] and ideal MHD [9] up to $4^{\text {th }}$-order accuracy. Details about the extension to axisymmetric geometries can be found in [5]. To control unphysical Gibbs oscillations inherent to high-order hyperbolic methods, we use an evanescent hyperviscosity model that has been initially proposed in [10] for LES-type models. Practically, the idea is to consider system (10) with an additional right-hand side $\nabla \cdot(0, \underline{\boldsymbol{\tau}}, \underline{\boldsymbol{\tau}} \mathbf{u}-\mathbf{q}, 0,0)^{T}$ where the strain rate tensor $\underline{\boldsymbol{\tau}}$ and heat flux $\mathbf{q}$ are given by:

$$
\underline{\boldsymbol{\tau}}=\nu\left[\nabla \mathbf{u}+(\nabla \mathbf{u})^{\mathrm{T}}\right]+\left(\beta-\frac{2}{3} \nu\right)(\nabla \cdot \mathbf{u}) \underline{\mathbf{I}} \quad \text { and } \quad \mathbf{q}=-\chi_{e} \nabla T_{e}-\chi_{i} \nabla T_{i} .
$$

The physical constants $\beta$ and $\nu$ respectively denote the bulk and shear viscosities whereas $\chi_{e}$ and $\chi_{i}$ denote the electronic and ionic thermal conductivities. To preserve consistency with the initial hyperbolic system, these constants are replaced by evanescent artificial terms $\beta^{*}, \nu^{*}, \chi_{e}^{*}$ and $\chi_{i}^{*}$ that vanish when the space step $\Delta=\sqrt{\Delta r \Delta z}$ tends to zero. In this study, the $\beta^{*}$ and $\chi_{\alpha}^{*}$ terms follow the hyperviscosity model given in [10], that is, with $a$ the sound speed:

$$
\beta^{*}=C_{\beta} \cdot\left\langle\rho\left|\nabla^{4} \sqrt{\left|\nabla \mathbf{u}+(\nabla \mathbf{u})^{\mathrm{T}}\right|^{2}}\right|\right\rangle \cdot \Delta^{6} \quad \text { and } \quad \chi_{\alpha}^{*}=C_{\chi_{\alpha}} \cdot\left\langle\frac{\rho a}{T_{\alpha}}\left|\nabla^{4} \epsilon_{\alpha}\right|\right\rangle \cdot \Delta^{5} \text {, }
$$


the $\langle\cdot\rangle$ operator denoting a 2-D Gaussian filter. The $\nu^{*}$ term is chosen to maximize the viscous strain rate tensor without breaking the CFL condition of the hyperbolic scheme. In order to prevent it from introducing too much diffusion near shock waves, it is also filtered using a smoothed Heavyside function. Finally, the $\nu^{*}$ term writes:

$$
\nu^{*}=C_{\nu} \cdot F(\nabla \cdot \mathbf{u}) \cdot(\rho c) \Delta \quad \text { where } \quad F(x)= \begin{cases}1 & \text { if } x \geq 0 \\ \exp \left(-400\left(\frac{x}{\max |x|}\right)^{2}\right) & \text { if } x \leq 0\end{cases}
$$

Constants $C_{\beta}, C_{\nu}$ and $C_{\chi_{\alpha}}$ are user-defined. Practically, the $\beta^{*}$ and $\chi_{\alpha}^{*}$ terms tend to reduce oscillations respectively near shock waves (as shown in [8] for example) and contact discontinuities, whereas $\nu^{*}$ has been designed to preserve the sphericity of the flow (see Figure 4 in section 4 which illustrates the improvements provided by (11) regarding sphericity).

\subsection{Parabolic operators: thermal and resistive conductivity}

\subsubsection{Thermal diffusion}

Using (1), thermal diffusion equations write:

$$
\begin{aligned}
\partial_{t}\left(\rho_{i} C_{v_{i}} T_{i}\right)+\nabla \cdot \mathbf{q}_{\mathbf{i}} & =-3 \frac{m_{e}}{m_{i}} n_{e} \nu_{e i} k_{B}\left(T_{i}-T_{e}\right), \\
\partial_{t}\left(\rho_{e} C_{v_{e}} T_{e}\right)+\nabla \cdot \mathbf{q}_{\mathbf{e}} & =-3 \frac{m_{e}}{m_{i}} n_{e} \nu_{e i} k_{B}\left(T_{e}-T_{i}\right) .
\end{aligned}
$$

Both equations being very close, we will only focus here on (13). The semi-implicit time scheme we propose is:

$$
\rho_{e} C_{v_{e}} \frac{T_{e}^{n+1}-T_{e}^{n}}{\Delta t}-\nabla \cdot\left(k_{B}\left({\overline{\overline{\kappa_{e}}}}^{n} \cdot \nabla T_{e}^{n+1}\right)+\frac{k_{B}(\overline{\bar{\beta}} \cdot \mathbf{J})^{n}}{e} T_{e}^{n+1}\right)=-3 \frac{k_{B} m_{e}}{m_{i}} \cdot n_{e}^{n} \cdot \nu_{e i}^{n} \cdot\left(T_{e}^{n+1}-T_{i}^{n+1}\right)
$$

Taking $\frac{1}{\Delta r \Delta z} \int(14) r d r d z$ over $\left[z_{i-\frac{1}{2}} ; z_{i+\frac{1}{2}}\right] \times\left[r_{j-\frac{1}{2}} ; r_{j+\frac{1}{2}}\right]$ one gets the following finite volume type scheme:

$$
\begin{array}{r}
r_{j} C_{v_{e}}\left(\rho_{e}\right)_{i, j}^{n}\left(\left(T_{e}\right)_{i, j}^{n+1}-\left(T_{e}\right)_{i, j}^{n}\right)+\frac{r_{j} k_{B} \Delta t}{\Delta z}\left(q_{i+\frac{1}{2}, j}^{*}-q_{i-\frac{1}{2}, j}^{*}\right)+\frac{k_{B} \Delta t}{\Delta r}\left(r_{j+\frac{1}{2}} q_{i, j+\frac{1}{2}}^{*}-r_{j-\frac{1}{2}} q_{i, j-\frac{1}{2}}^{*}\right) \\
=-3 \Delta t \frac{k_{B} m_{e}}{m_{i}} \cdot\left(n_{e}\right)_{i, j}^{n} \cdot\left(\nu_{e i}\right)_{i, j}^{n} \cdot\left(\left(T_{e}\right)_{i, j}^{n+1}-\left(T_{i}\right)_{i, j}^{n+1}\right)
\end{array}
$$

with fluxes along the $z$ and $r$-directions being respectively given by:

$$
\begin{aligned}
q_{i+\frac{1}{2}, j}^{*}= & -\left(\frac{\left(\kappa_{e}^{\perp}\right)_{i, j}^{n}+\left(\kappa_{e}^{\perp}\right)_{i+1, j}^{n}}{2}\right) \cdot\left(\frac{\left(T_{e}\right)_{i+1, j}^{n+1}-\left(T_{e}\right)_{i, j}^{n+1}}{\Delta z}\right)-\left(\frac{\overline{\bar{\beta}} \cdot \mathbf{J})_{i, j}^{n}+(\overline{\bar{\beta}} \cdot \mathbf{J})_{i+1, j}^{n}}{2 e}\right) \cdot\left(\frac{\left(T_{e}\right)_{i+1, j}^{n+1}+\left(T_{e}\right)_{i, j}^{n+1}}{2}\right) \\
& +\left(\frac{\left(\kappa_{e}^{\wedge}\right)_{i, j}^{n}+\left(\kappa_{e}^{\wedge}\right)_{i+1, j}^{n}}{2}\right) \cdot\left(\frac{\left(T_{e}\right)_{i+1, j+1}^{n+1}+\left(T_{e}\right)_{i, j+1}^{n+1}-\left(T_{e}\right)_{i+1, j-1}^{n+1}-\left(T_{e}\right)_{i, j-1}^{n+1}}{4 \Delta r}\right), \\
q_{i, j+\frac{1}{2}}^{*}= & -\left(\frac{\left(\kappa_{e}^{\perp}\right)_{i, j}^{n}+\left(\kappa_{e}^{\perp}\right)_{i, j+1}^{n}}{2}\right) \cdot\left(\frac{\left(T_{e}\right)_{i, j+1}^{n+1}-\left(T_{e}\right)_{i, j}^{n+1}}{\Delta r}\right)-\left(\frac{(\overline{\bar{\beta}} \cdot \mathbf{J})_{i, j}^{n}+(\overline{\bar{\beta}} \cdot \mathbf{J})_{i, j+1}^{n}}{2 e}\right) \cdot\left(\frac{\left(T_{e}\right)_{i, j+1}^{n+1}+\left(T_{e}\right)_{i, j}^{n+1}}{2}\right) \\
& -\left(\frac{\left(\kappa_{e}^{\wedge}\right)_{i, j}^{n}+\left(\kappa_{e}^{\wedge}\right)_{i, j+1}^{n}}{2}\right) \cdot\left(\frac{\left(T_{e}\right)_{i+1, j}^{n+1}+\left(T_{e}\right)_{i+1, j+1}^{n+1}-\left(T_{e}\right)_{i-1, j}^{n+1}-\left(T_{e}\right)_{i-1, j+1}^{n+1}}{4 \Delta z}\right) .
\end{aligned}
$$


Together with the similar scheme for the ionic temperature, solving (12-13) therefore leads to solve a linear system $A x=b$ in the unknowns $\left(T_{e}, T_{i}\right)_{i, j}^{n+1}$. In the general case, $A$ is a non-symmetric matrix, and the linear system is solved using a biconjugate gradient algorithm. One also has the

Proposition 2.1. If $B=0$, the matrix $A$ is symmetric positive definite.

The proof, detailed in [5], is rather easy since $\mathbf{J}, \kappa_{e}^{\wedge}$ and $\kappa_{i}^{\wedge}$ are zero in the $B=0$ case.

\subsubsection{Resistive diffusion}

The resistive diffusion system of equations writes, with $\mathbf{J}=\frac{c}{\mu} \nabla \times \mathbf{B}$ according to (2):

$$
\begin{aligned}
\partial_{t} \mathbf{B}+c \nabla \times(\overline{\bar{\rho}} \cdot \mathbf{J}) & =0, \\
\partial_{t}\left(\rho_{e} \epsilon_{e}\right)-(\overline{\bar{\rho}} \cdot \mathbf{J}) \cdot \mathbf{J} & =0 .
\end{aligned}
$$

Note that both equations are decoupled. We first solve (15) with the semi-implicit time scheme:

$$
\frac{B^{n+1}-B^{n}}{\Delta t}+\frac{c^{2}}{\mu} \nabla \times\left(\overline{\bar{\rho}}^{n} \cdot\left(\nabla \times \mathbf{B}^{n+1}\right)\right)=0
$$

Taking $\frac{1}{\Delta r \Delta z} \int(17) d r d z$ over the cell $\left[z_{i-\frac{1}{2}} ; z_{i+\frac{1}{2}}\right] \times\left[r_{j-\frac{1}{2}} ; r_{j+\frac{1}{2}}\right]$ one gets the following finite volume type scheme:

$$
B_{i, j}^{n+1}-B_{i, j}^{n}=\frac{c^{2} \Delta t}{\mu \Delta z}\left(R_{i+\frac{1}{2}, j}^{*}-R_{i-\frac{1}{2}, j}^{*}\right)-\frac{c^{2} \Delta t}{\mu \Delta r}\left(R_{i, j+\frac{1}{2}}^{*}-R_{i, j-\frac{1}{2}}^{*}\right)
$$

with fluxes along the $z$ and $r$-directions being respectively given by:

$$
\begin{aligned}
R_{i+\frac{1}{2}, j}^{*}= & \left(\frac{\left(\rho^{\perp}\right)_{i, j}^{n}+\left(\rho^{\perp}\right)_{i+1, j}^{n}}{2}\right) \cdot\left(\frac{B_{i+1, j}^{n+1}-B_{i, j}^{n+1}}{\Delta z}\right) \\
& -\left(\frac{\left(\rho^{\wedge}\right)_{i, j}^{n}+\left(\rho^{\wedge}\right)_{i+1, j}^{n}}{2}\right) \cdot\left(\frac{r_{j+1}\left(B_{i+1, j+1}^{n+1}+B_{i, j+1}^{n+1}\right)-r_{j-1}\left(B_{i+1, j-1}^{n+1}+B_{i, j-1}^{n+1}\right)}{4 r_{j} \Delta r}\right), \\
R_{i, j+\frac{1}{2}}^{*}= & \left(\left(\rho^{\perp}\right)_{i, j}^{n}+\left(\rho^{\perp}\right)_{i, j+1}^{n}\right) \cdot\left(\frac{r_{j+1} B_{i, j+1}^{n+1}-r_{j} B_{i, j}^{n+1}}{\left(r_{j}+r_{j+1}\right) \Delta r}\right) \\
& -\left(\frac{\left(\rho^{\wedge}\right)_{i, j}^{n}+\left(\rho^{\wedge}\right)_{i, j+1}^{n}}{2}\right) \cdot\left(\frac{B_{i+1, j+1}^{n+1}+B_{i+1, j}^{n+1}-B_{i-1, j+1}^{n+1}-B_{i-1, j}^{n+1}}{4 \Delta z}\right) .
\end{aligned}
$$

Hereagain, we are led to solve a linear system $A x=b$ in the unknowns $(B)_{i, j}^{n+1}, A$ being a non-symmetric matrix. A biconjugate gradient algorithm is therefore used. Once the magnetic field has been updated, (16) is solved by

$$
\left(\rho_{e} \epsilon_{e}\right)_{i, j}^{n+1}-\left(\rho_{e} \epsilon_{e}\right)_{i, j}^{n}=\Delta t\left[\left(\overline{\bar{\rho}}^{n} \cdot \mathbf{J}^{n+1}\right) \cdot \mathbf{J}^{n+1}\right]_{i, j} .
$$

\subsection{Self-generated magnetic field source term}

As said in section 1, Hall and Nernst effects are not considered in this work. The source terms system therefore writes:

$$
\partial_{t} \mathbf{B}-c \nabla \times \mathbf{S}_{\text {self }}=0,
$$

with $\mathbf{S}_{\text {self }}$ given by (9). Due to this term, self-generated magnetic field terms will appear (only along the $\theta$ direction in the case of 2-D axisymmetric geometries) as soon as the density and pressure gradients are not 
aligned. Indeed

$$
\nabla \times\left(\frac{1}{n_{e}} \nabla p_{e}\right)=-\frac{1}{n_{e}^{2}} \nabla n_{e} \wedge \nabla p_{e}
$$

An easy way to numerically preserve this property consists in discretizing the magnetic field equation using (19) as it is done in [11]. In the specific case $\mathbf{B}=B \mathbf{e}_{\theta}$, it leads to the following scheme:

$$
B_{i, j}^{n+1}-B_{i, j}^{n}=\frac{c \Delta t}{e\left[\left(n_{e}\right)_{i, j}^{n}\right]^{2}}\left[\left(d_{r} n_{e}\right)_{i, j} \cdot\left(d_{z} p_{e}\right)_{i, j}-\left(d_{z} n_{e}\right)_{i, j} \cdot\left(d_{r} p_{e}\right)_{i, j}\right] \quad \text { with } \quad\left\{\begin{array}{l}
\left(d_{z} \phi\right)_{i, j}=\frac{\phi_{i+1, j}^{n}-\phi_{i-1, j}^{n}}{2 \Delta z} \\
\left(d_{r} \phi\right)_{i, j}=\frac{\phi_{i, j+1}^{n}-\phi_{i, j-1}^{n}}{2 \Delta r}
\end{array}\right.
$$

One could also prefer a conservative approach: taking $\frac{1}{\Delta r \Delta z} \int(18) d r d z$ over $\left[z_{i-\frac{1}{2}} ; z_{i+\frac{1}{2}}\right] \times\left[r_{j-\frac{1}{2}} ; r_{j+\frac{1}{2}}\right]$, one gets the following finite volume type scheme:

$$
B_{i, j}^{n+1}-B_{i, j}^{n}=\frac{c \Delta t}{2 \Delta z}\left(S_{i+1, j}^{r, n}-S_{i-1, j}^{r, n}\right)-\frac{c \Delta t}{2 \Delta r}\left(S_{i, j+1}^{z, n}-S_{i, j-1}^{z, n}\right) \text { with }\left\{\begin{array}{l}
S_{i, j}^{r, n}=\left(\frac{1}{e n_{e}} \partial_{r} p_{e}\right)_{i, j}^{n}=\frac{1}{e\left(n_{e}\right)_{i, j}^{n}}\left(d_{r} p_{e}\right)_{i, j} \\
S_{i, j}^{z, n}=\left(\frac{1}{e n_{e}} \partial_{z} p_{e}\right)_{i, j}^{n}=\frac{1}{e\left(n_{e}\right)_{i, j}^{n}}\left(d_{z} p_{e}\right)_{i, j} .
\end{array}\right.
$$

Practically, due to numerical errors, both schemes actually generate magnetic field terms of equivalent magnitudes in presence of colinear density and pressure gradients. We therefore chose to implement the magnetic field conservative one given by (20).

\section{VAlidation test PROBLEMS FOR THE DifFUSION OPERATOR}

\subsection{Non-linear diffusion test problem}

The first validation test problem concerns the non-linear diffusion equation in spherically symmetric geometry $\partial_{t} T-\nabla \cdot\left(\nu T^{\nu-1} \nabla T\right)=0$, together with the initial condition $T(r, t=0)=\left(1-r^{2}\right)^{\frac{1}{\nu-1}}$ and homogeneous Neumann boundary conditions, for which a solution is given by:

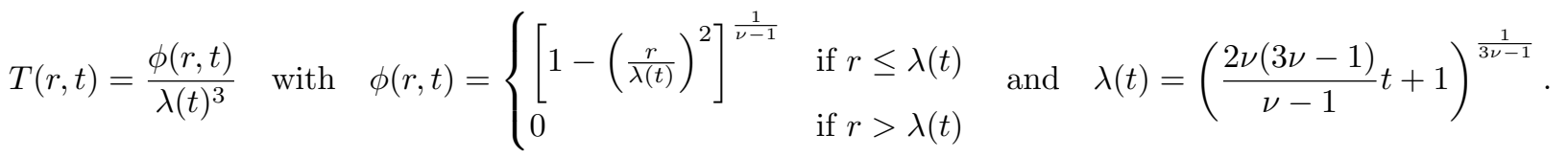

We set the parameter $\nu$ to 3.5 and have run computations with our 2-D axisymmetric diffusion scheme on different meshes until $t=0.1$. Figure 1 plots the approximate solution obtained on 100 cells at $t=0.1$. Some error measurements between the exact and numerical solutions have been performed on the smooth region $r \in[0 ; 1]$ using a $L^{1}$ norm in space and time. Table 2 summarizes these results and the experimental order of convergence which corresponds to the theoretical order. Second-order accuracy could be achieved using a more accurate time scheme but this goes beyond the scope of this paper. 


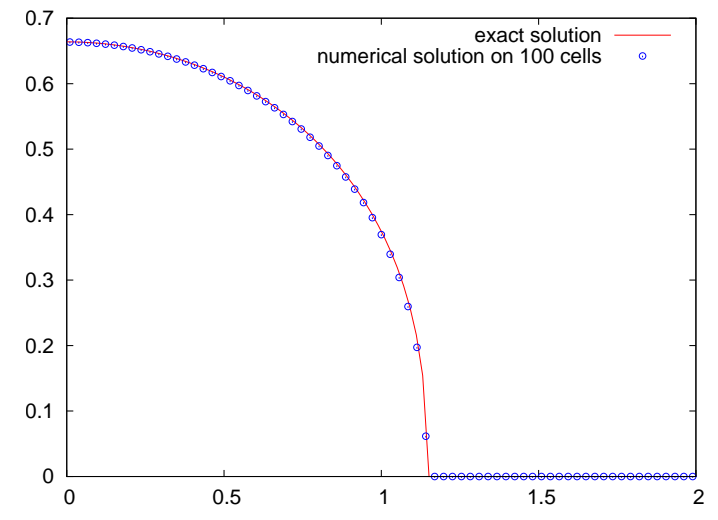

Figure 1. Exact and numerical solution (slice along the $z=r$ axis) on 100 cells at time $t=0.1$.

\begin{tabular}{|c|c|c|}
\hline Number of cells & Error & Order \\
\hline $50 \times 50$ & $4.56 \mathrm{e}-05$ & \\
$100 \times 100$ & $2.26 \mathrm{e}-05$ & 1.01 \\
$200 \times 200$ & $1.13 \mathrm{e}-05$ & 1.00 \\
$400 \times 400$ & $5.72 \mathrm{e}-06$ & 0.99 \\
$800 \times 800$ & $2.87 \mathrm{e}-06$ & 0.99 \\
\hline
\end{tabular}

TABLE 2. Error between the analytical and numerical solutions measured using a $L^{1}$ norm on the $(r, t) \in$ $[0 ; 1] \times[0 ; 0.1]$ space-time domain.

\subsection{Anisotropic diffusion test problem (in planar geometry)}

The second validation test problem, taken from [12], concerns the anisotropic diffusion equation in planar geometry:

$$
\partial_{t} u-\nabla \cdot(\kappa(u) \nabla u)=0 \quad \text { with } \quad \kappa(u)=\left(\begin{array}{cc}
\kappa_{1}(x, y) & \kappa_{2}(x, y) u \\
-\kappa_{2}(x, y) u & \kappa_{1}(x, y)
\end{array}\right)
$$

The test problem is set on the $[0 ; 1]^{2}$ square with following initial conditions:

$$
u(t=0)= \begin{cases}-1 & \text { if } y \geq 0.5 \\ 0 & \text { if } y<0.5\end{cases}
$$

and boundary conditions:

$$
\begin{aligned}
u=-1 & \text { on the } y=1 \text { boundary, } \\
\nabla u \cdot \vec{\nu}=0 & \text { on other boundaries. }
\end{aligned}
$$

The $\kappa_{1}$ and $\kappa_{2}$ values are given by:

$$
\left(\kappa_{1}, \kappa_{2}\right)= \begin{cases}\left(10^{-2}, 10^{-1}\right) & \text { if } x \leq 0.5 \\ \left(10^{-3}, 10^{-5}\right) & \text { if } x>0.5\end{cases}
$$

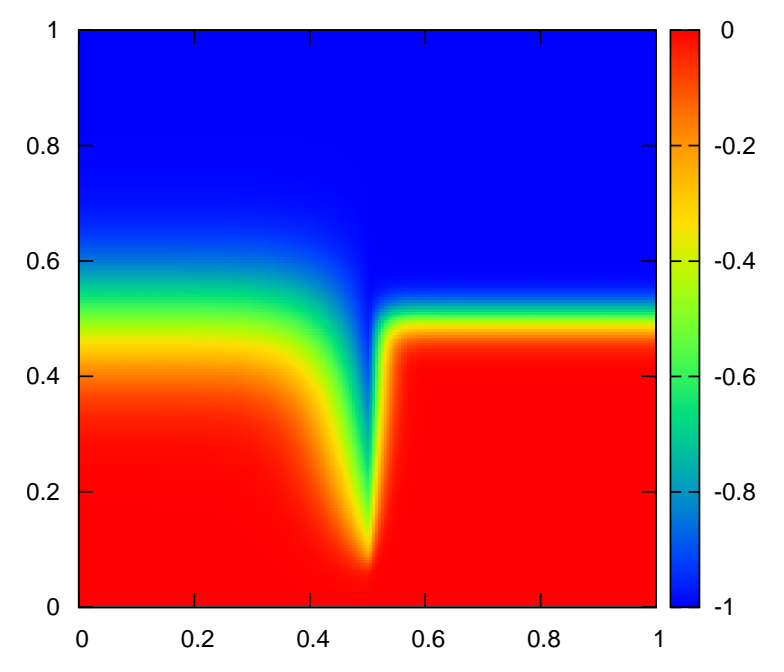

Figure 2. Solution at time $t=0.5$ for the anisotropic diffusion test problem.

The $\kappa$ tensor being non-symmetric, this problem is solved using a biconjugate gradient algorithm. Figure 2 plots the solution obtained at time $t=0.5$ on $200 \times 200$ cells with our diffusion scheme. Results are similar to those presented in the literature. 


\section{Direct DRIVE ICF DECELERATION PHASE BENCHMARK}

The test problem studied in this section is taken from [2]. Initial conditions given below correspond to the state of a spherically symmetric ICF shell of deuterium-tritium (DT) at the beginning of the deceleration phase. Both electrons and ions $\left(Z_{i}=1\right)$ follow a perfect gas law with $\gamma_{e, i}=5 / 3$. The plasma is assumed to be fully ionized and the average mass of DT is set to $\frac{3}{2} m_{p}$, where $m_{p}$ is the mass of a proton. Closure relations are given in [1] and collision frequencies are computed as in [13].

\subsection{Initial conditions (in CGS units)}

The considered domain is $400 \mu m$ wide and divided in four regions.

- For $r \leq 0.018 \mathrm{~cm}$ :

$u^{(1)}(r)=-6.01121 \cdot 10^{8} r-8.59652 \cdot 10^{11} r^{2}+9.00161 \cdot 10^{13} r^{3}-2.55503 \cdot 10^{15} r^{4}$,

$p^{(1)}(r)=1.1364 \cdot 10^{14}-1.4741 \cdot 10^{15} r+2.5482 \cdot 10^{18} r^{2}-2.6914 \cdot 10^{20} r^{3}+8.3432 \cdot 10^{21} r^{4}$,

$\rho^{(1)}(r)=p^{(1)}(r) \cdot 2.586759 \cdot 10^{-15} \cdot\left(1-1.7226 \cdot 10^{4} r^{2.56061}\right)^{-1}$,

$p_{e}^{(1)}(r)=p^{(1)}(r) \cdot\left(-3.00077 \cdot 10^{20} r+9.44575 \cdot 10^{18}\right) \cdot\left(-2.02242 \cdot 10^{22} r^{2}-1.00185 \cdot 10^{21} r+3.3336 \cdot 10^{19}\right)^{-1}$.

- For $0.018 \mathrm{~cm}<r \leq 0.021 \mathrm{~cm}$ :

$u^{(2)}(r)=-1.176725 \cdot 10^{11}+2.40071 \cdot 10^{13} r-1.83229 \cdot 10^{15} r^{2}+6.19924 \cdot 10^{16} r^{3}-7.84614 \cdot 10^{17} r^{4}$,

$p^{(2)}(r)=-9.90683 \cdot 10^{17}+2.04304 \cdot 10^{20} r-1.57663 \cdot 10^{22} r^{2}+5.39767 \cdot 10^{23} r^{3}-6.91788 \cdot 10^{24} r^{4}$,

$\rho^{(2)}(r)=0.251956-1.99589 \cdot 10^{4} r^{3}+\left(97.3135-4.61145 \cdot 10^{3} r\right)^{-1}$,

$p_{e}^{(2)}(r)=\left(-3.812829 \cdot 10^{12} r^{5}+3.758976 \cdot 10^{11} r^{4}-1.481416 \cdot 10^{10} r^{3}+2.916974 \cdot 10^{8} r^{2}-2.869374 \cdot 10^{6} r+1.127981 \cdot 10^{4}\right) \cdot p^{(2)}(r)$.

- For $0.021 \mathrm{~cm}<r \leq 0.0313 \mathrm{~cm}$ :

$u^{(3)}(r)=2.4198 \cdot 10^{9}-3.73623 \cdot 10^{11} r+2.10716 \cdot 10^{13} r^{2}-5.24689 \cdot 10^{14} r^{3}+4.88108 \cdot 10^{15} r^{4}$,

$p^{(3)}(r)=5.5673 \cdot 10^{16}-8.3122 \cdot 10^{18} r+4.5695 \cdot 10^{20} r^{2}-1.0932 \cdot 10^{22} r^{3}+9.619 \cdot 10^{22} r^{4}$,

$\rho^{(3)}(r)=270.52-4.3198 \cdot 10^{4} r+2.3752 \cdot 10^{6} r^{2}-5.17461 \cdot 10^{7} r^{3}+3.6722 \cdot 10^{8} r^{4}$,

$p_{e}^{(3)}(r)=0.5 \cdot p^{(3)}(r)$.

- For $0.0313 \mathrm{~cm}<r \leq 0.04 \mathrm{~cm}$ :

$u^{(4)}(r)=\min \left(u^{(3)}(r), 0\right)$,

$\rho^{(4)}(r)=\exp \left(-1.20269 \cdot 10^{3}(r-0.0313)\right)$,

$p^{(4)}(r)=2.443754 \cdot 10^{13} \rho^{(4)}(r)$,

$p_{e}^{(4)}(r)=\left(p_{e}^{(3)} / \rho_{e}^{(3)}\right)(r=0.0313) \cdot \rho_{e}^{(4)}(r)$.

The Rayleigh-Taylor unstable deceleration phase of this spherically symmetric ICF implosion is here simulated with our 2-D code in axisymmetric geometry. The computational domain is a $400 \mu m$ square in the $(z, r)$ plane with wall boundary conditions along the $z$ and $r$ axis and open boundary conditions elsewhere.

\subsection{One-temperature ICF benchmark: numerical results}

In order to validate our diffusion scheme and the underlying physical model, 2-D axisymmetric simulations are compared to converged 1-D results obtained with the one-temperature LPC code used in [2] in spherical 
Density
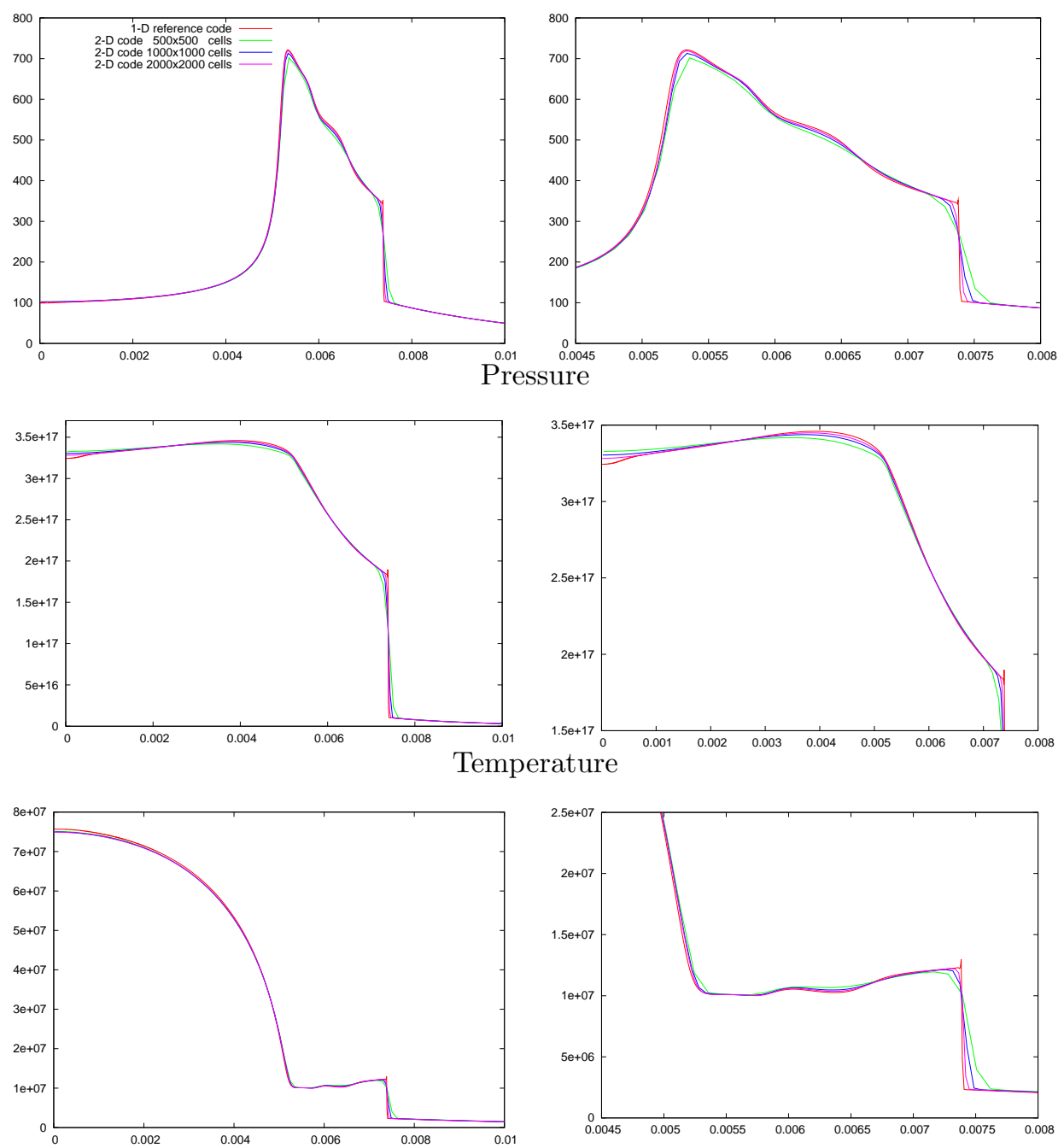

Figure 3. One-temperature ICF test problem. Comparison between 1-D Lagrangian LPC [2] results and 2-D simulations (slice along the $z=r$ direction) at time $t=600 \mathrm{ps}$.

geometry. The test problem is initialized as explained in section 4.1 but both ionic and electronic temperatures are here computed using the following identity:

$$
T_{i}=T_{e}=\frac{p}{\rho C_{v}(\gamma-1)}
$$

with $C_{v}=6.471255 \cdot 10^{7}$ and $\gamma=5 / 3$. The hyperbolic and thermal diffusion systems are the only one considered here, so that no magnetic field will appear. The hyperbolic part is solved using third-order Lagrange-remap fluxes and a first-order Godunov dimensional splitting. The CFL number is set to 0.7 and evanescent hyperviscosity constants are $C_{\beta}=C_{\nu}=0.8$ and $C_{\chi}=0$. Figure 3 presents the results obtained with both codes at time $t=600 \mathrm{ps}$. It shows a good agreement between both simulations, the results of the 2-D axisymmetric code apparently converging to those of the 1-D LPC reference code. 

$\mathbf{C}_{\nu}=\mathbf{0}$
$\mathrm{C}_{\nu}=\mathbf{0 . 4}$
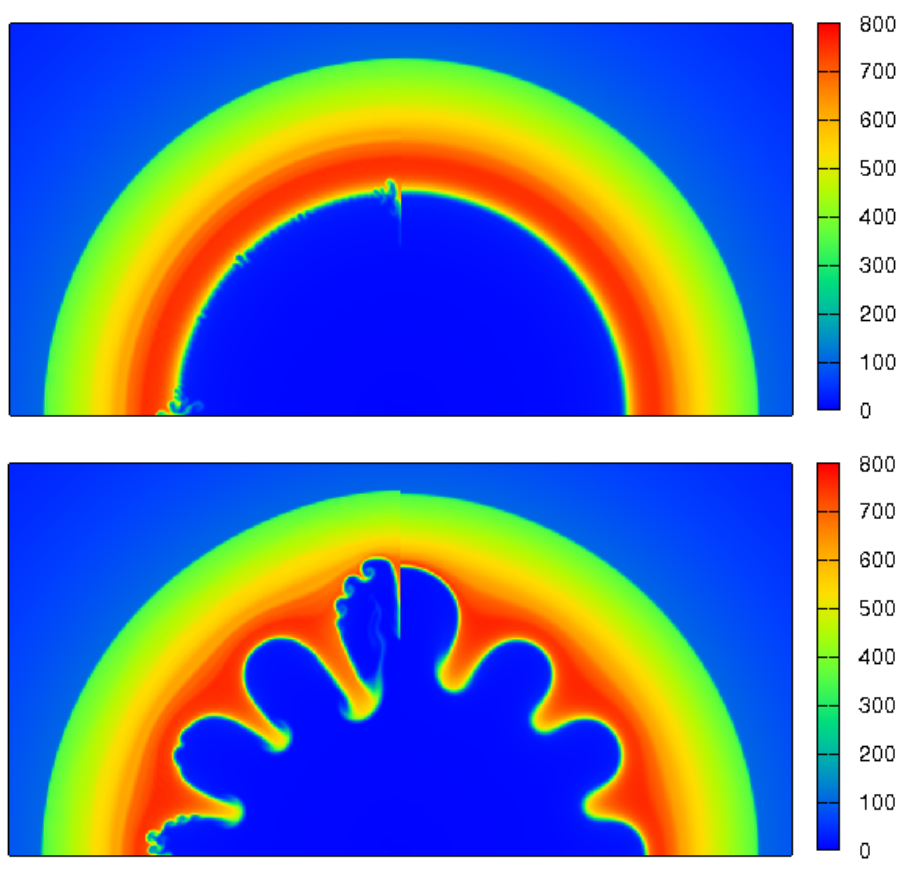

$\mathbf{C}_{\nu}=\mathbf{0}$

$\mathrm{C}_{\nu}=\mathbf{0 . 4}$

Figure 4. Effect of evanescent hyperviscosity $\nu^{*}$ coefficient on the two-temperature ICF test problem at time $t=600 \mathrm{ps}$ ns (zoom on the $[0 ; 0.008]^{2}$ square). Hydrodynamics without heat conduction, with $\beta^{*}=0.8$ and $\kappa^{*}=0.05$. Density plot on $2000^{2} / 4000^{2}$ cells (resp. left $/$ right column), for $A_{0}=0$ and $0.5 \mu \mathrm{m}$.

\subsection{Two-temperature ICF benchmark: numerical results}

We now consider the two-temperature case, where $C_{v_{e, i}}$ are given by (1). In order to emulate the slight gap to sphericity that is caused by the non-uniformity of the laser irradiation and/or shell rugosity, initial conditions are slightly perturbed along the radial direction as in [2]: let

$$
r^{\varepsilon}(r)=r+A_{l}(r) P_{l}(\cos \theta) \quad \text { with } \quad A_{l}(r)=A_{0} \exp \left(-l\left|\frac{r}{r_{i}}-1\right|\right)
$$

where $A_{0}$ is the initial perturbation amplitude, $r_{i}=210 \mu \mathrm{m}$ the location of the gas/shell interface, $l$ the mode number and $P_{l}$ the Legendre polynomial with $\theta$ the angle between the radial and the symmetry axis. Perturbed initial conditions $U^{\varepsilon}$ are then defined by $U^{\varepsilon}\left(r^{\varepsilon}(r)\right)=U(r)$ with $U(r)$ given in section 4.1.

In order to illustrate the effect of the evanescent hyperviscosity $\nu^{*}$ coefficient (11), we first propose a pure hydrodynamics test. In Figure 4 we compare simulations on two different meshes for $A_{0}=0$ and $0.5 \mu m$ run with $C_{\nu}=0$ and 0.4. Symmetry preservation is improved and mesh effects are clearly reduced when this term is activated.

In the second test, we compare simulations on the whole model with/without the self-generated magnetic field source term for different initial perturbation amplitudes. Figure 5 shows that the perturbations growth 

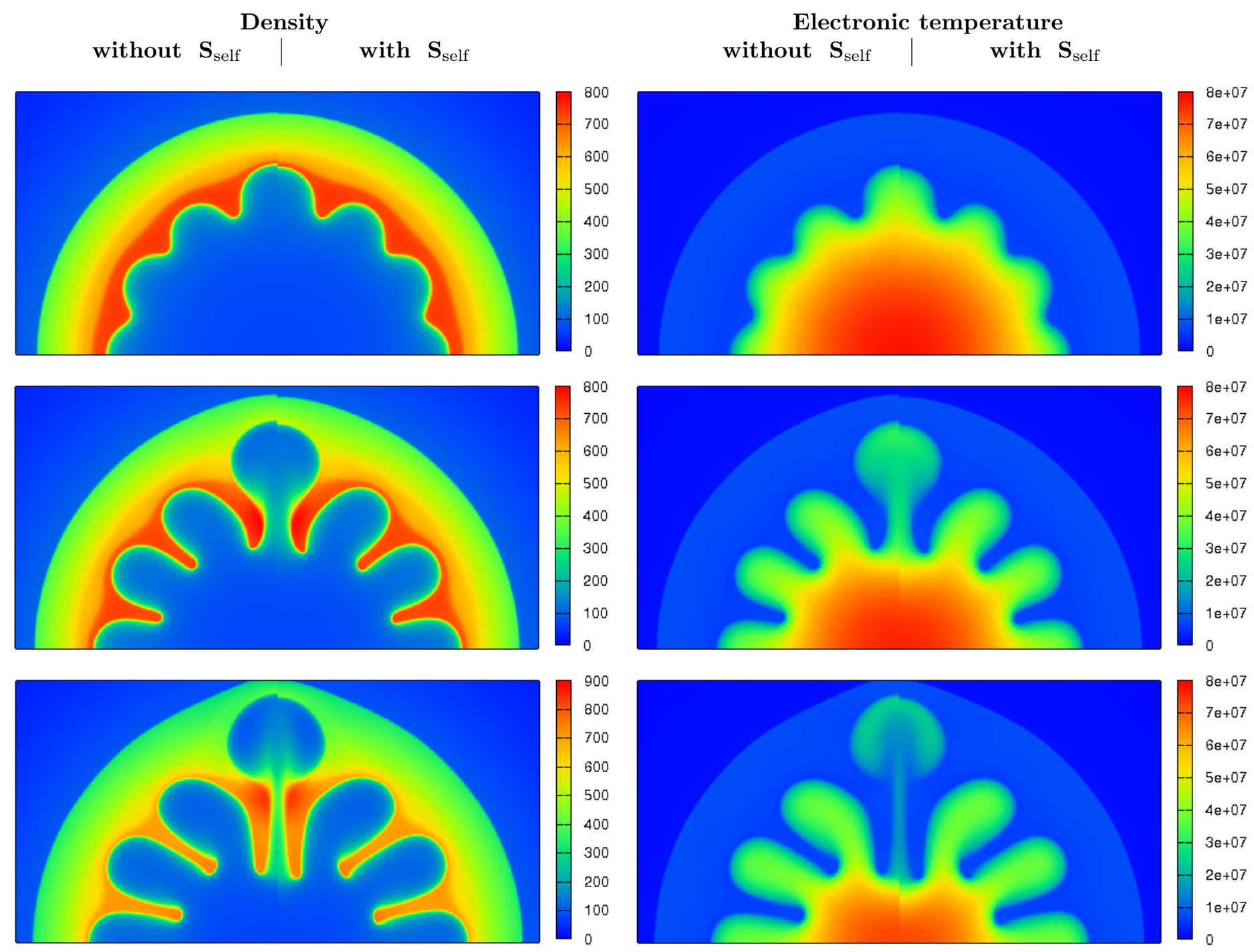

Figure 5. Two-temperature ICF test problem at time $t=600 \mathrm{ps}$ (zoom on the $[0 ; 0.008]^{2}$ square). Comparison with/without self-generated magnetic field term. From top to bottom: $A_{0}=1,5$ and $10 \mu m$.

rates are slightly reduced with the $B$ field, but the latter does not seem to radically modify the topology of the flow.

Convergence studies on the whole model have been performed for the $A_{0}=5 \mu \mathrm{m}$ case. Figure 6 reports results obtained on three increasing mesh sizes which show that the perturbation amplitudes still evolves. Detailed studies on the hydrodynamics case have indeed shown that finer meshes were required if one wants to compute converged perturbation growth rates (see [5] for further details). Nevertheless it can be seen that the magnitude and the distribution of different quantities does not roughly change when the mesh is refined. Convergence in $L^{1}$ norm of the magnetic field can also be seen on Figure 7 (right). 
Density, magnetic field and electronic temperature plots are given in Figure 8 for different initial perturbation amplitudes. Note that without any perturbations, the flow is supposed to be spherically symmetric so that density and pressure gradients are colinear. The rotational of $\mathbf{S}_{\text {self }}$ is therefore zero and no magnetic field should be generated according to (18). For $A_{0}=0$ (top of Figure 8), the magnetic field which appears is therefore only due to numerical approximations. Nevertheless, various simulations have shown that, when the mesh is refined, its amplitude is reduced and $\|B\|_{1} \rightarrow 0$ (see Figure 7 - right). As expected, magnetic fields are generated around both sides of spikes where the electronic temperature gradient is the steepest. Figure 8 also shows that the higher the initial perturbation amplitude is, the higher the magnitude of the B field. This can also be seen on Figure 7 (left). For $A_{0}=5 \mu \mathrm{m}$, we therefore compute a magnetic field about $30 M G$, a value given in [14].

\section{Conclusions And Perspectives}

We have presented numerical methods for solving the two-temperature resistive MHD equations with the self-generated magnetic field term. The complete system has been split into several subsystems according to the nature of the underlying mathematical operator. It allowed us to build and validate numerical schemes for each of these sets of equations. Contrarily to works generally published on this topic (see [11] for instance), the hyperbolic scheme is conservative and formulated in total energy. Whereas techniques developed for this subsystem have been studied in previous papers $[5,8,9]$, we have shown here the good behavior of our schemes for the parabolic operators on both isotropic and anisotropic diffusion test problems as well as on a direct drive ICF deceleration benchmark.

On one hand, we have seen on the latter that the generated magnetic field for the non-perturbated case, which is only due to numerical approximations, is reduced when the mesh is refined and its $L^{1}$-norm tends to zero with the grid size. On the other hand, numerical results with a non-zero initial perturbation seem convincing and are in accordance with published works (see [14] for instance): magnetic fields are generated around both sides of spikes of Rayleigh-Taylor modes, reach a magnitude of about $30 \mathrm{MG}$ depending on the initial perturbation amplitude and slightly reduce growth rates compared to classical two-temperature simulations.

Ongoing work mainly concerns the accurate estimation of instabilities growth rates which have already been studied in the case of hydrodynamics with and without thermal diffusion in [2]. We aim to provide informations on the influence of magnetic field on these growth rates. To that end, we still have to add Hall and Nernst effects and to improve HPC capabilities of our code. It currently allows us to perform computations on fine meshes (up to $4000 \times 4000$ cells here, i.e. about 100 million unknowns) but we would like to get closer to convergence.

\section{REFERENCES}

[1] S.I. Braginskii. Transport processes in a plasma. In M.A. Leontovich, editor, Reviews of plasma physics. Consultants Bureau - New-York, 1965.

[2] M. Temporal, S. Jaouen, L. Masse, and B. Canaud. Hydrodynamic instabilities in ablative tamped flows. Phys. of Plasmas, $13,2006$.

[3] K. Mima, T. Tajima, and J.-N. LeBoeuf. Magnetic field generation by the Rayleigh-Taylor instability. Phys. Rev. Let., 41(25):1715-1719, 1978.

[4] S. Jaouen. A purely Lagrangian method for computing linearly-perturbed flows in spherical geometry. J. Comp. Phys., 198:80$105,2007$.

[5] M. Wolff. Analyse mathmatique et numrique du systme de la MHD rsistive avec termes de champ magntique auto-gnr. PhD thesis, Universit de Strasbourg, 2011. To be published.

[6] F. Coquel and C. Marmignon. Numerical methods for weakly ionized gases. Astrophys. Space Sci., 260:15-27, 1998.

[7] F. Coquel and C. Marmignon. A Roe-type linearization for the Euler equations for weakly ionized gases. Hermes Sci. Publ., Paris, 1999. 
$500^{2}$ cells
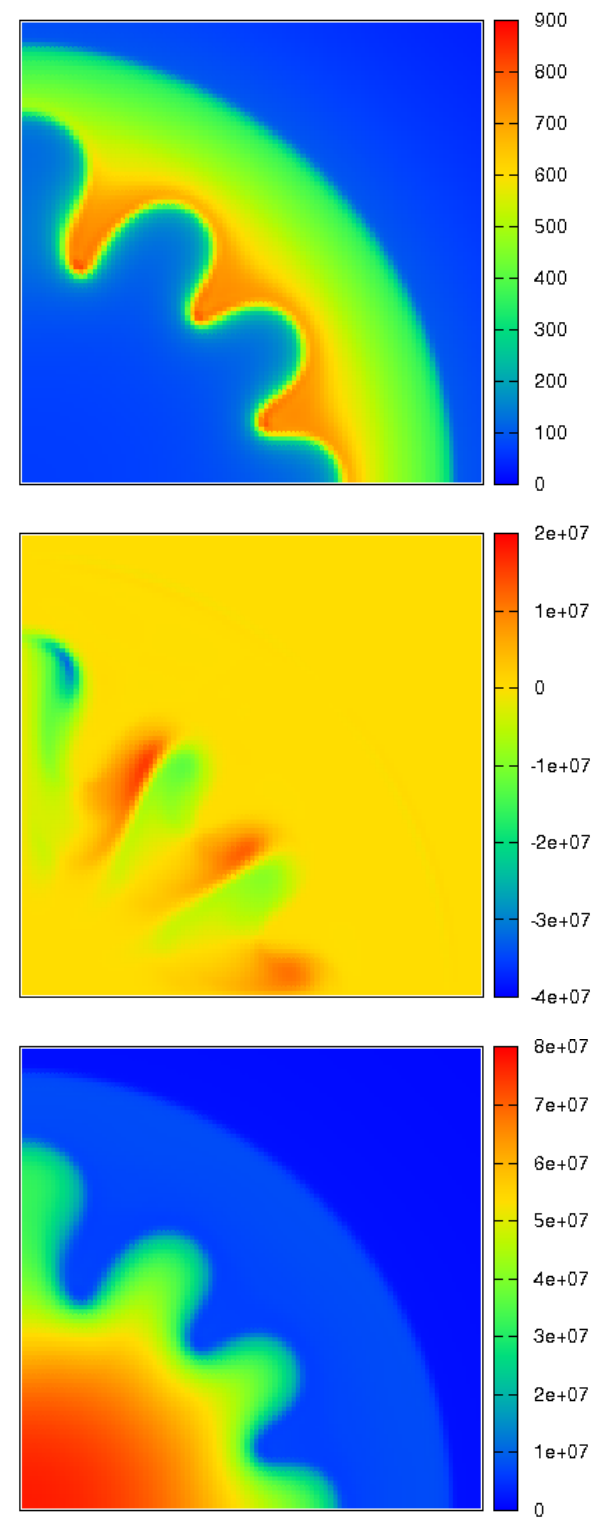

$1000^{2}$ cells
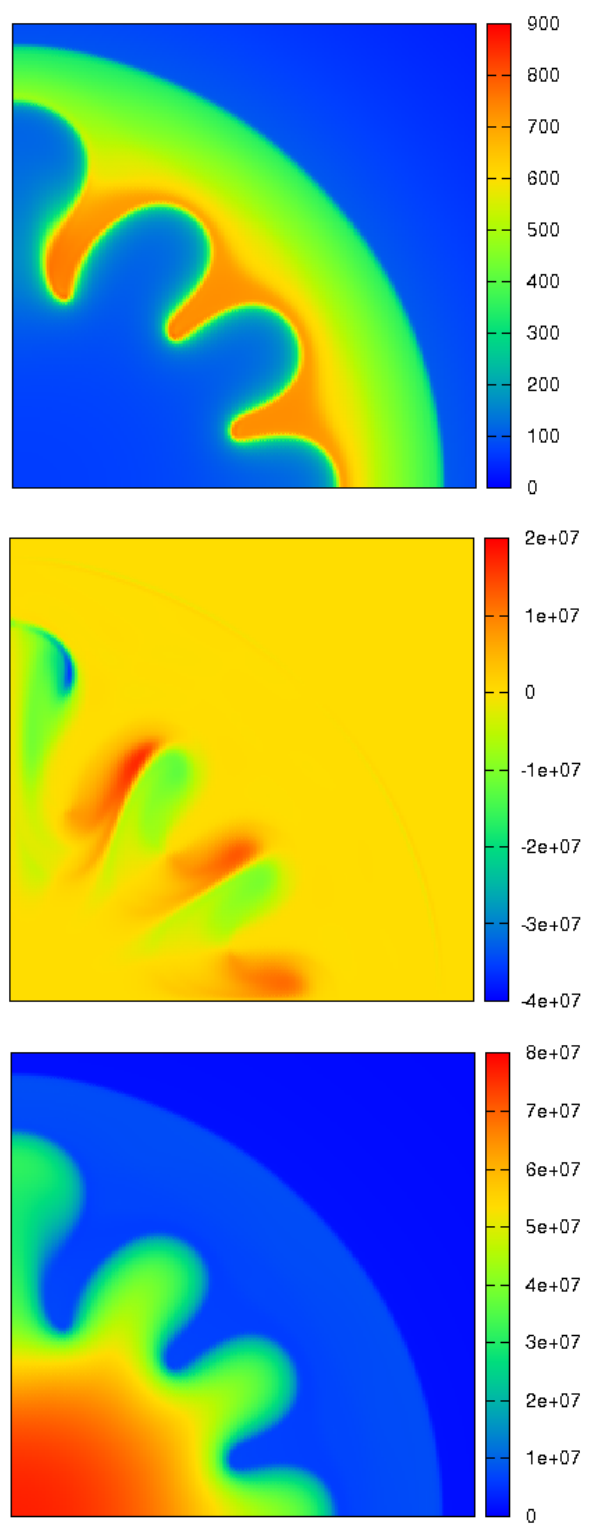

\section{$2000^{2}$ cells}
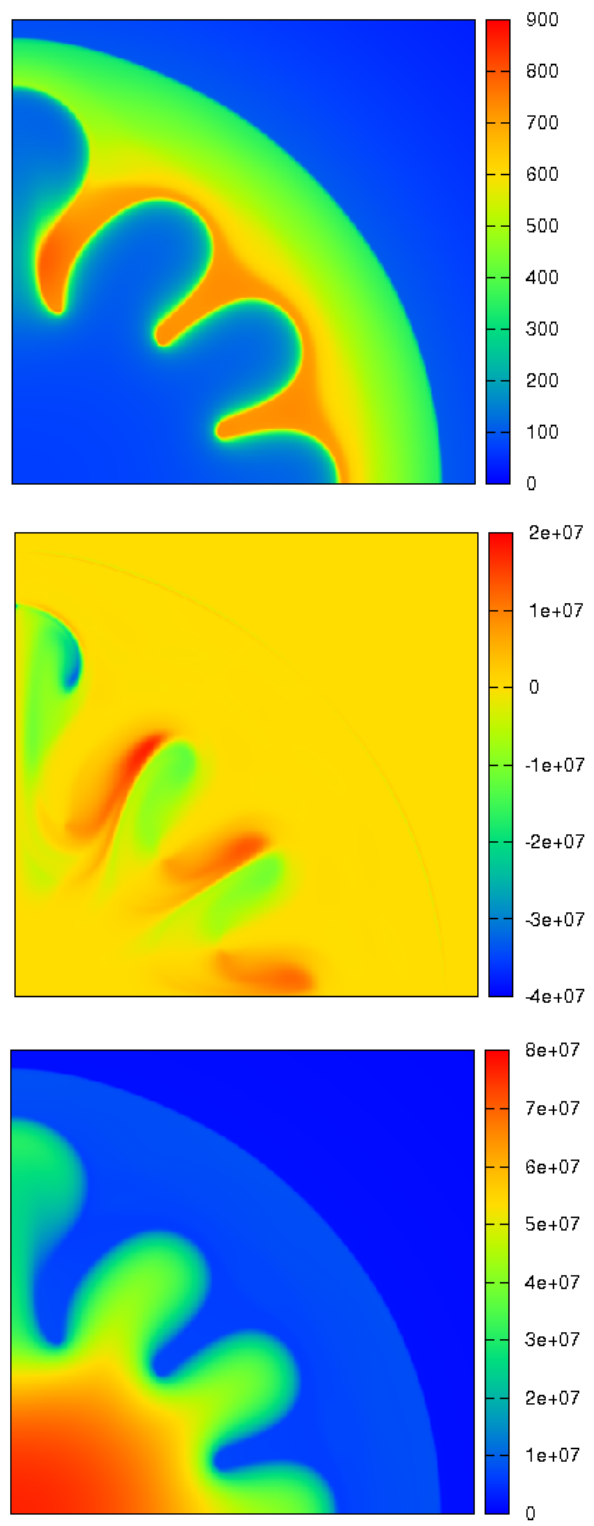

FiguRE 6. Two-temperature ICF test problem at time $t=600 \mathrm{ps}$ for $A_{0}=5 \mu \mathrm{m}$ (zoom on the $[0 ; 0.008]^{2}$ square). From top to bottom: density, magnetic field, electronic temperature.

[8] F. Duboc, C. Enaux, S. Jaouen, H. Jourdren, and M. Wolff. High-order dimensionally split Lagrange-remap schemes for compressible hydrodynamics. C.R. Acad. Sci. Paris, 348:105-110, 2010.

[9] M. Wolff, S. Jaouen, and H. Jourdren. High-order dimensionally split Lagrange-remap schemes for ideal magnetohydrodynamics. In Discrete and Continuous Dynamical Systems Series S: proceedings of Numerical Models for Controlled Fusion (NMCF'09), Porquerolles, France, 2009.

[10] A. Cook. Artificial fluid properties for large-eddy simulation of compressible turbulent mixing. Phys. of Fluids, $19,2007$.

[11] R.S. Bowers and J.R. Wilson. Numerical modeling in applied physics and astrophysics. Jones and Barlett Publishers, Boston, 1991. 

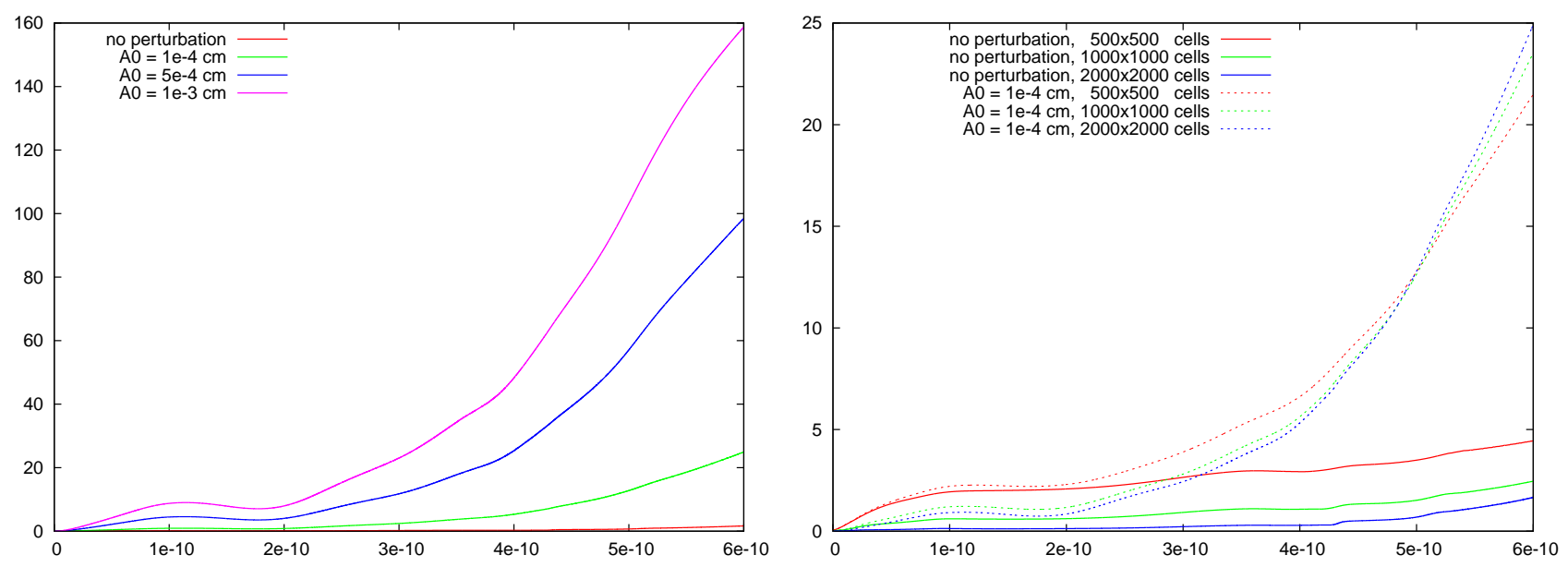

FiguRE 7. Two-temperature ICF test problem, $\|B\|_{1}$ versus time. Left: varying the initial perturbation amplitude $A_{0}=0,1,5$ and $10 \mu m$ on a $2000 \times 2000$ mesh. Right: varying the mesh size for $A_{0}=0$ and $1 \mu \mathrm{m}$.

[12] F. Hermeline. A finite volume method for the approximation of diffusion operators on distorted meshes. J. Comp. Phys., 160:481-499, 2000.

[13] A. Decoster. Fluid equations and transport coefficient of plasmas. In P.-A. Raviart, editor, Modelling of collisions, Research in applied mathematics, pages 1-137. Masson, Paris, 1997.

[14] A. Hata, K. Mima, A. Sunahara, H. Nagatomo, and A. Nishiguchi. Dynamics of self-generated magnetic fields in stagnation phase and their effects on hot spark formation. Plasmas and Fusion Research, 1(20):1-6, 2006. 


\section{Density}
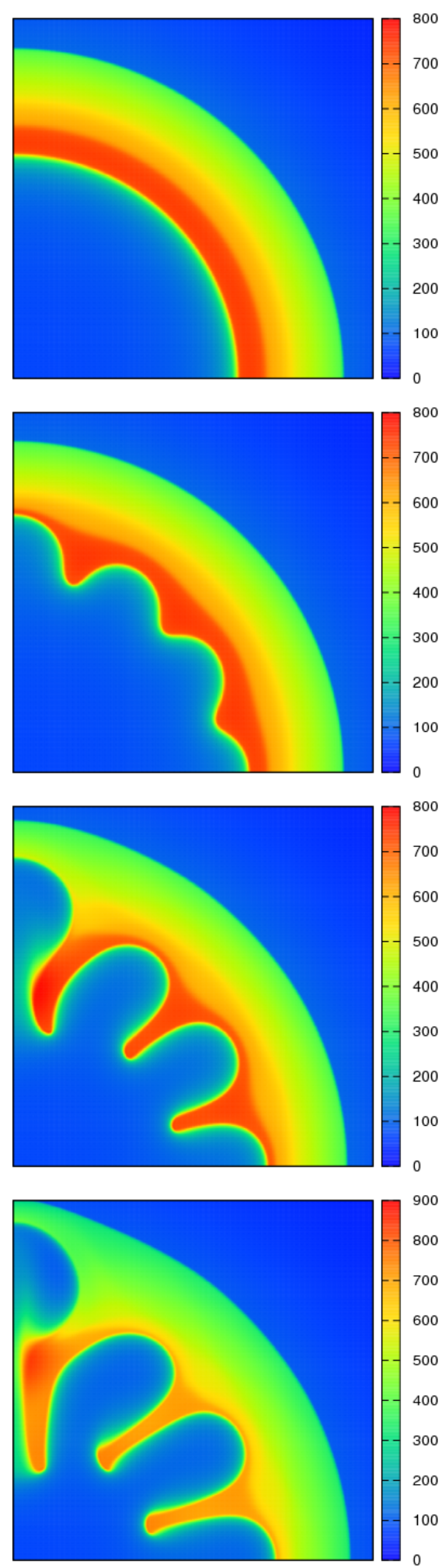

Magnetic field
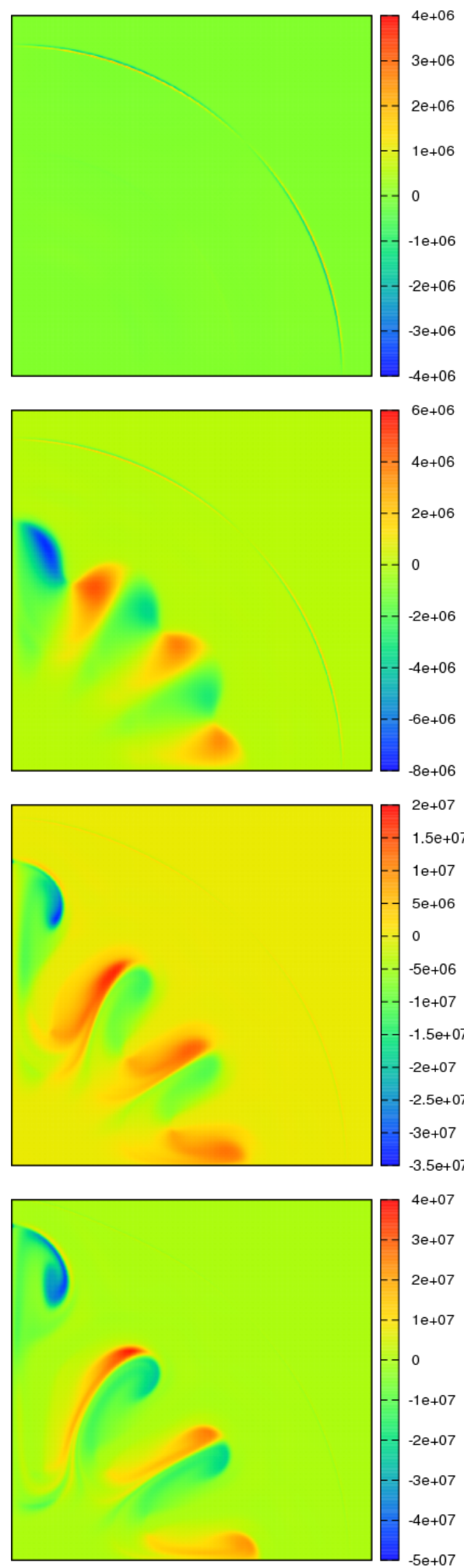

Electronic temperature
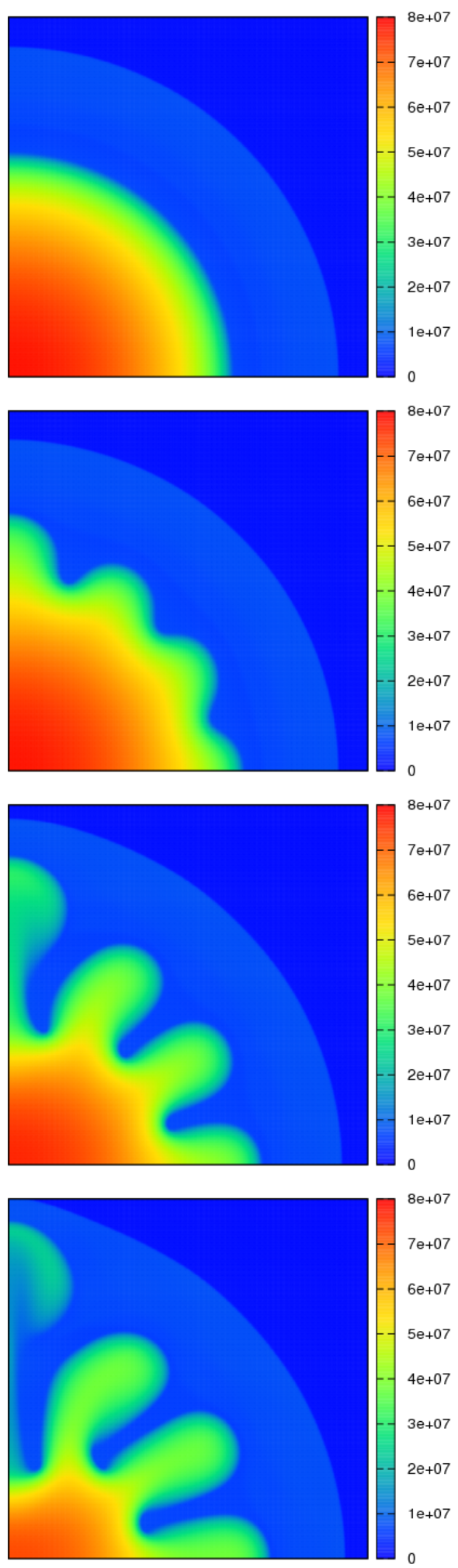

Figure 8. Two-temperature ICF test problem at time $t=600 \mathrm{ps}$ (zoom on the $[0 ; 0.008]^{2}$ square). From top to bottom: $A_{0}=0,1,5$ and $10 \mu \mathrm{m}$. 